\title{
Photochemistry and magnetic resonance spectroscopy as probes of supramolecular structures and migration pathways of organic molecules and radicals adsorbed on zeolites
}

\author{
Nicholas J. Turro, ${ }^{* a} \dagger$ Xuegong Lei, ${ }^{a}$ Wei Li, ${ }^{a}$ Ann McDermott, ${ }^{a}$ Lloyd Abrams, ${ }^{b}$ M. Francesca Ottavianai ${ }^{c}$ and \\ Hege Støgård Beard $d$ \\ a Chemistry Department, Columbia University, New York, NY 10027, USA \\ ${ }^{b}$ E. I. duPont de Nemours and Co., Central Research Department, Experimental Station, Wilmington, DE 19880, USA \\ c University of Florence, Department of Physical Chemistry, Via Gino Capponi, 9, 50121 Florence, Italy \\ ${ }^{d}$ Chemistry Department, University of Oslo, Oslo, Norway
}

Magnetic resonance, surface area measurements and computational techniques have been integrated to elucidate the supramolecular photochemistry of two isomeric ketones adsorbed on two MFI zeolites (silicalite and ZSM-5) and to demonstrate that common factors proportional to the available external surface area operate to determine the measured parameters in each case.

We report here examples of supramolecular photochemistry ${ }^{1,2}$ of ketones adsorbed on molecular sieve zeolites (possessing MFI topology). The systems employed are two isomeric ketones, $o \mathrm{ACOB}$ and $p \mathrm{ACOB}$, whose molecular photochemistries are essentially identical, ${ }^{3}$ but whose supramolecular photochemistries $^{4}$ (Scheme 1) are a strong function of supramolecular composition, structure and dynamics.<smiles>[Y][I-]1=CC=C(CC(=O)Cc2ccc(C)cc2)C=C1</smiles>

$p A C O B \quad X=\mathrm{H}$

$\left[{ }^{2} H_{5}\right]-p A C O B X=D$

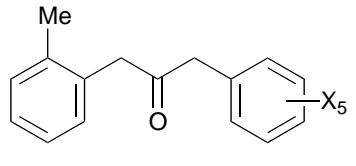

$O A C O B X=H$

$\left[{ }^{2} \mathrm{H}_{5}\right]-O A C O B X=D$
As shown in Scheme 1, the external surface of ZSF-5 zeolites ${ }^{5}$ possesses two sites for binding of $o$ ACOB molecules: (i) the holes on the external surface ( $c a .30 \%$ of the external

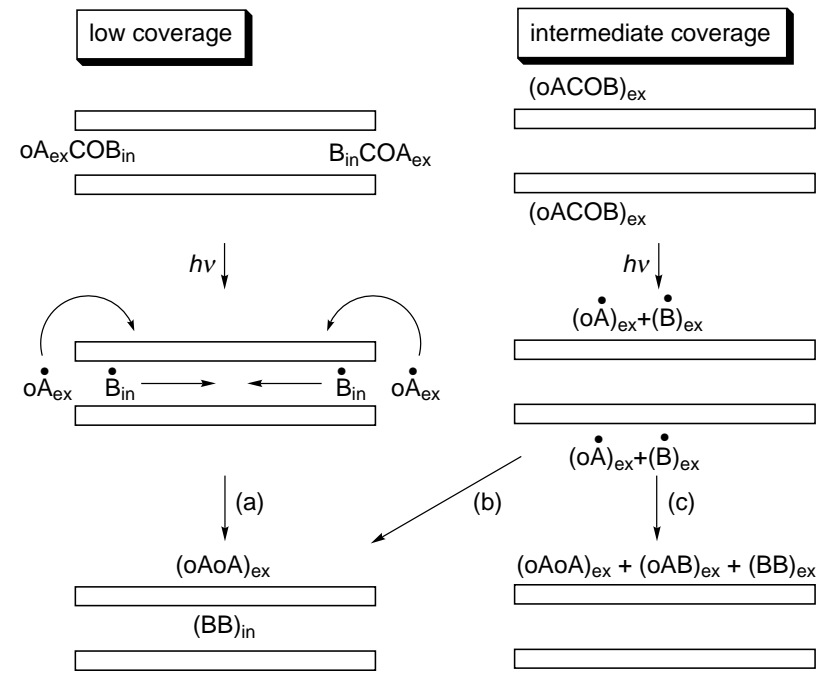

Scheme 1 Schematic description of the supramolecular photochemistry of $o \mathrm{MeDBK} / \mathrm{MFI}$ complexes and $p \mathrm{MeDBK} / \mathrm{MFI}$ complexes at loadings corresponding to less than a monolayer. The holes are filled first (left) and then the solid framework. surface area) and (ii) the remaining 'solid framework' of the external surface ( $\mathrm{ca} .70 \%$ of the external surface area). As ketone is loaded, a monolayer of ketone will form at some point and loading of ketone beyond this point will cause the formation of multilayers.

According to the paradigm 4 of Scheme 1, the products of the photochemistry of $o$ ACOB adsorbed on MFI zeolites will depend both on the initial siting of the ketone and on the dynamics of the radicals produced by photolysis of $o$ ACOB adsorbed at these sitings.

The photolyses of $o \mathrm{ACOB}$ and $p \mathrm{ACOB}$ adsorbed on two MFI zeolites, ${ }^{5}$ silicalite (surface area $=5 \mathrm{~m}^{2} \mathrm{~g}^{-1}$ ) ${ }^{6}$ and ZSM-5 (surface area $\left.=16 \mathrm{~m}^{2} \mathrm{~g}^{-1}\right),{ }^{6}$ were investigated as a function of loading of ketone in the range $c a$. 0.1 to $c a$. $5 \%$ w/w. Fig. 1 summarizes the results of the 'cage effect'7 as a function of loading. The product mixture from the photolysis of the $p \mathrm{ACOB} /$ zeolite sysem was found to exhibit a strong positive cage effect $(\mathrm{CE})^{7}$ over the entire loading range investigated for both silicalite and ZSM-5. In contrast, the product mixture from the photolysis of the $o \mathrm{ACOB} / z e o l i t e$ system depends strongly on the loading, exhibiting a strong negative cage effect ${ }^{7}$ at low loadings, and an increasingly positive cage effect ${ }^{7}$ reaching a
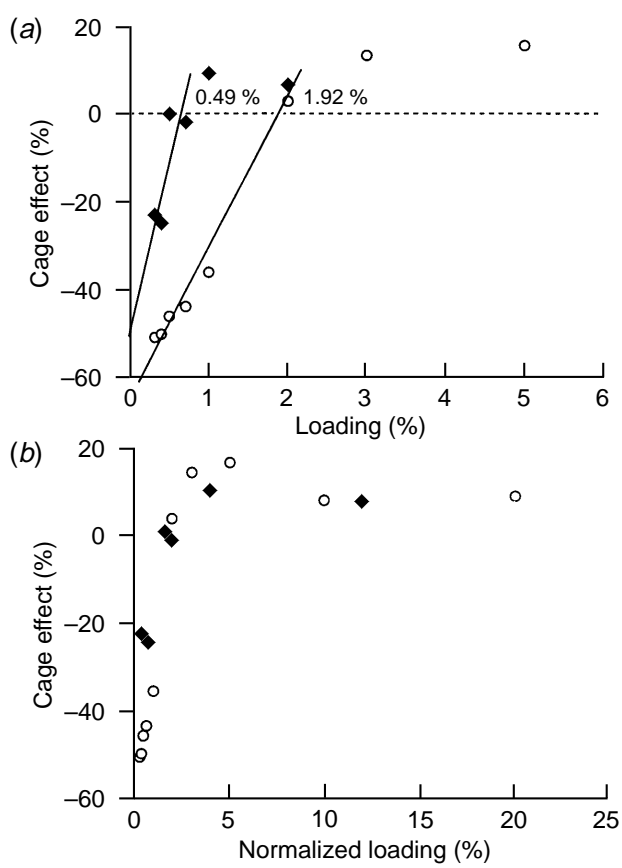

Fig. 1 ( $a$ ) Loading and $(b)$ normalized loading dependence of the cage effect for the photolysis of $o$ MeDBK on $(\bigcirc) 25 \mathrm{M}-5$ and $(>)$ silicalite zeolites 

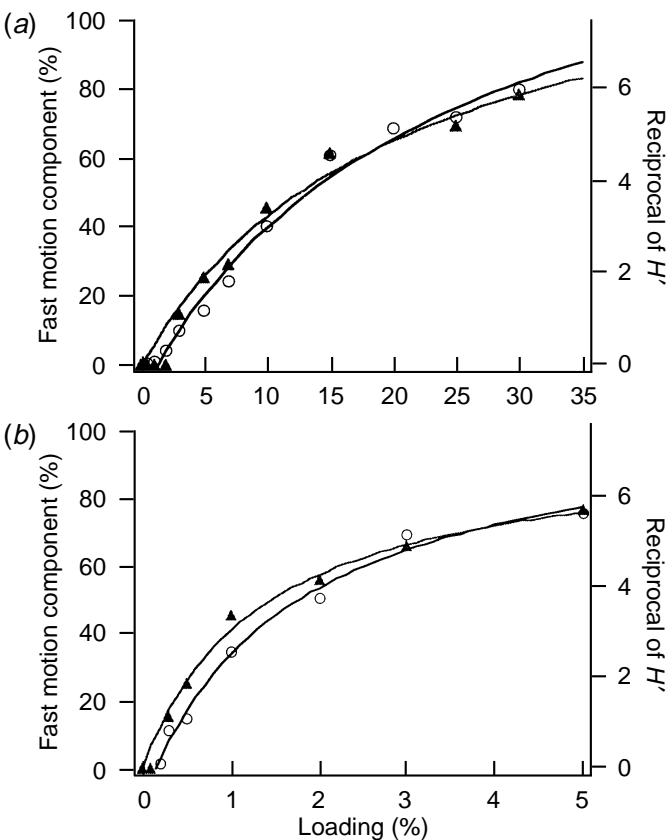

Fig. 2 Values for $(a)$ ZSM-5 and $(b)$ silicalite zeolites of $(\bigcirc)$ the reciprocal of $H^{\prime}$ from ${ }^{2} \mathrm{H}$ NMR spectra and $(\boldsymbol{\Delta})$ the fast motion component extracted from EPR spectra, as a function of loading of $o \mathrm{ACOB}$

maximum and progressing towards a limiting cage effect of zero at the highest loadings [Fig. 1(a)].

For low loadings (conditions for which the surface area and number of holes determine the $\mathrm{CE}$ ), a linear fit of the data in Fig. 1 yields values of 0.49 and $1.92 \%$ for the loadings at which a CE of $c a$. $0 \%$ is achieved (assumed to correspond approximately to the formation of the monolayer). The ratio of these loadings is 3.9 and is strikingly similar to the observed ratio $(3: 2)$ of the experimentally measured surface areas of the two zeolite samples. ${ }^{6}$ These results demonstrate that the external surface area is a key parameter in understanding the results.

The reciprocal of the half width of the signal $\left(H^{\prime}=1 / \Delta H_{1 / 2}\right)$ in the solid state ${ }^{2} \mathrm{H}$ NMR of $\left[{ }^{2} \mathrm{H}_{5}\right]-o A C O B$ was determined as a function of loading (Fig. 2). The values of $H^{\prime}$ are related to the motion $^{8}$ of ring B. If the ring is plugged in a hole on the external surface, its motion is expected to be more constrained than when it is adsorbed on the solid external surface. ${ }^{8}$ Finally, if the ring is in a liquid-like multilayer, the motion is expected to be effectively isotropic and unconstrained.

For the lowest loadings, the values of $H^{\prime}$ for both zeolite samples are small (motion constrained) and constant, as expected if the ketones first occupy the holes on the external surface. The value of $H^{\prime}$ begins to increase, and then plateau as expected if the ketones begin to occupy a liquid-like environment.

The form of the graph of the ${ }^{2} \mathrm{H}$ NMR data in Fig. 2 may be described by a Langmuir expression such as eqn. (1), where $Y$ is

$$
Y=M(k) x /(1+k x)
$$

the value of a parameter proportional to coverage, $x$ is the loading of $o$ ACOB $(\mathrm{w} / \mathrm{w}), M$ is the maximum value of $Y$ [computed from fitting the data to eqn. (1)], and $k$ is related to the equilibrium constant for adsorption of the ketone on the external surface.

If we assume that the motion of the adsorbed $o$ MeDBK molecules is directly related to the external surface coverage, then the reciprocal of the half-widths ${ }^{8}$ of the ${ }^{2} \mathrm{H}$ NMR data serves as parameter $Y$ in eqn. (1). At loadings below $0.3 \%$ the ${ }^{2} \mathrm{H}$ NMR signal is relatively broad and constant, implying that the first $o \mathrm{ACOB}$ molecules adsorbed are more tightly bound to the holes than subsequently adsorbed molecules which bind to the solid external surface. ${ }^{9}$ However, for values of $H^{\prime}$ plotted as a function of loadings greater than $c a .1 \%$, the data fit eqn. (1) within experimental error (solid curves in Fig. 2). Values of $k=0.041 \pm 0.010$ and $0.56 \pm 0.50$ for ZSM-5 and silicalite, respectively, are evaluated from the fit to eqn. (1). The data for both zeolite samples overlap within experimental error if the data for silicalite are scaled by a factor of $c a$. 5, which is of the order of the ratio of the measured external surface area of the two zeolites and the ratio of the linear portions of the slopes of the photochemical data in Fig. 1(a).

A second experiment employing EPR spectroscopy confirms indirectly the results of the photochemical and ${ }^{2} \mathrm{H}$ NMR experiments. In these experiments 4-oxo-TEMPO (T) was employed as an EPR probe of binding of $o$ ACOB to the external surface. A small amount $(0.1 \%)$ of $\mathrm{T}$ was added to the zeolite samples and the influence of the addition of $o$ ACOB on the EPR signal of the probe was observed. In the absence of $o \mathrm{ACOB}$, the EPR spectrum consists of only a broad signal characteristic peak, a strongly motion-constrained (slow component) probe bound to the external surface. Addition of ketone causes the conversion of the broad signal to a sharp, three line signal (fast component) characteristic of a mobile probe displaced by the ketone to a weaker binding region of the external surface. The resulting spectra could be simulated ${ }^{10}$ as the sum of a fast and slow component. Under the assumption that the ketone is displacing the constrained probe, the percent of fast component is proportional to the adsorbed ketone. Fig. 2 shows a plot of the percent of fast component as a function of loading for the two zeolites examined.

A plot of the percent of fast component as a function of loading fits eqn. (1) within the experimental error [Fig. 2(a)]. Values of $k=0.048 \pm 0.010$ and $0.76 \pm 0.11$ for ZSM-5 and silicalite, respectively, are evaluated from the fit to eqn. (1). Strikingly, these values are experimentally indistinguishable from the $k$ values evaluated from the ${ }^{2} \mathrm{H}$ NMR data $(k=0.041$ \pm 0.010 and $0.56 \pm 0.50$ for ZSM-5 and silicalite, respectively). Furthermore, the EPR data in Fig. 2 for the two zeolites overlap the data for the ${ }^{2} \mathrm{H}$ NMR data of Fig. 2 when the data for silicalite are scaled by the same factor ( $\mathrm{ca} .5)$ which corrects for the different surface areas of the two zeolites.

The authors at Columbia thank the National Science Foundation for its generous support of this research and Dr Zhi Liu for performing preliminary ${ }^{2} \mathrm{H}$ NMR experiments. X. G. L. and W. L. thank the Kanagawa Academy of Science and Technology for funding.

\section{Notes and References}

† E-mail: turro@chem.columbia.edu

1 Supramolecular Chemistry, J. M. Lehn, VCH, New York, 1995.

2 E. G. Derouane and Z. Gabelica, J. Catal., 1980, 65, 486.

3 N. J. Turro, M.-F. Chow, C.-J. Chung, G. Weed and B. Kraeutler, J. Am. Chem. Soc., 1980, 102, 4843 and references cited therein.

4 N. J. Turro, C. C. Cheng, L. Abrams and D. R. Corbin, J. Am. Chem. Soc., 1987, 109, 2449.

5 The external area available to a $o$ MeDBK molecule on the [010] MFI external surface was determined by computer simulation.

6 The surface area of the samples was determined by mercury porosimetry.

7 The cage effect is defined empirically in terms of the ratio of $o \mathrm{~A} o \mathrm{~A}$, $o \mathrm{AB}$ and $\mathrm{BB}$ measured experimentally (ref. 4).

8 R. J. Wittebort, E. T. Olejniczak and R. G. Griffin, J. Chem. Phys., 1987, 86, 5411 .

9 Adsorption of $o \mathrm{ACOB}$ on porous silica, a model for a flat version of the MFI external surface, results in only a sharp signal (ca. $5 \mathrm{kHz}$ ).

10 D. J. Schneider and J. H. Freed, in Biological Magnetic Resonance. Spin Labeling. Theory and Applications, ed. L. J. Berliner and J. Reuben, Plenum, New York, 1989, vol. 8, p. 1.

Received in Corvallis, OR, USA, 1st December 1997; 7/08688A 